Supporting Information for

\title{
Temperature-Induced Changes in Nanostructure of Hydrogels Based on Reversibly Cross-Linked Hyperbranched Polyglycidol with $\mathrm{B}(\mathrm{OH})_{4}{ }^{\ominus}$ Ions
}

\author{
Mateusz Gosecki†, Bozena Zgardzinska†, Monika Gosecka†* \\ $\dagger \quad$ Department of Engineering of Polymer Materials, Centre of Molecular and \\ Macromolecular Studies of Polish Academy of Sciences, ul. Sienkiewicza 112, 90-363 Lodz \\ *mdybko@,cbmm.lodz.pl; Telephone number: +48 426803235 \\ + Department of Nuclear Methods, Institute of Physics, Maria Curie-Sklodowska \\ University, Pl. M. Curie-Sklodowskiej 1, 20-031 Lublin, Poland.
}

\section{Table of Contents}

SI.1. Characteristics of hyperbranched polyglycidol. ..................................2

SI.1.1 GPC analysis of hyperbranched polyglycidol. ...........................2

SI.1.2 NMR analyses of hyperbranched polyglycidol. ............................

SI.2. Characteristics of hydrogel systems. ......................................

SI.2.1 Solid-state ${ }^{11}$ B NMR measurements of hydrogels at various temeperatures. ....5

SI.2.2 DSC analyses of hydrogels. .....................................6 
SI.1. Characteristics of hyperbranched polyglycidol.

SI.1.1 GPC analysis of hyperbranched polyglycidol

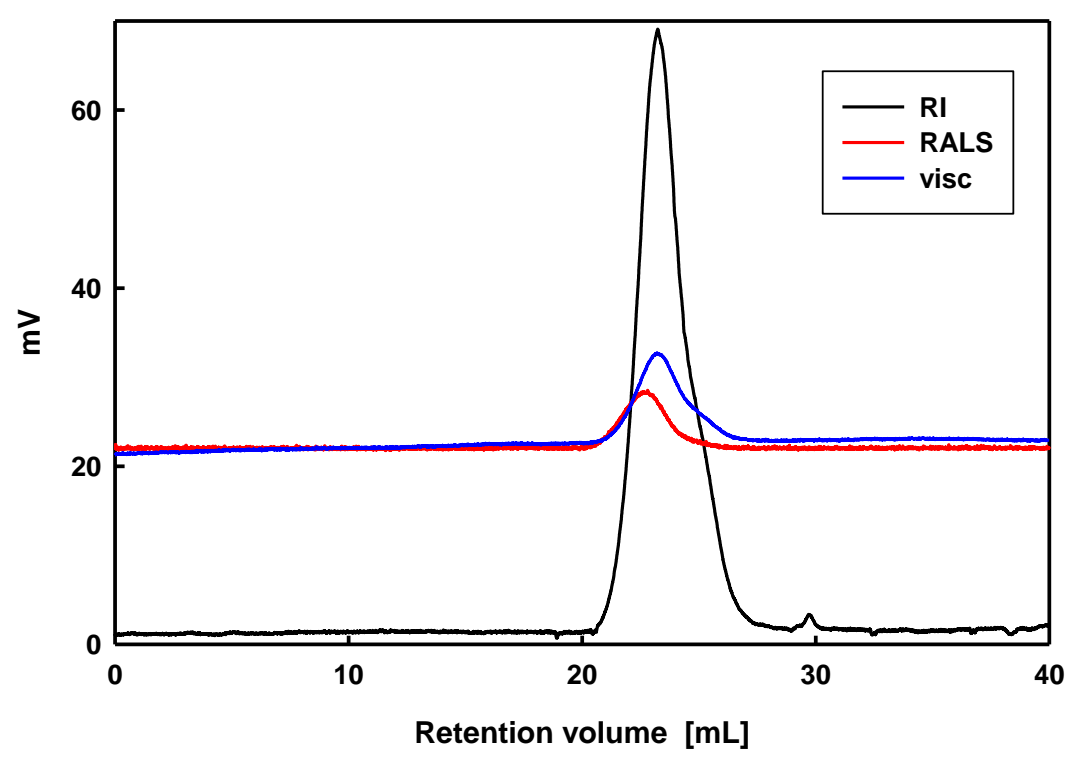

Figure S-1. GPC chromatograms of hyperbranched polyglycidol obtained from viscosimeter,

RI and RALS detectors. $\bar{M}_{n}=6430 ; \frac{\bar{M}_{w}}{M_{n}}=1,65$ 
SI.1.2. NMR analyses of hyperbranched polyglycidol

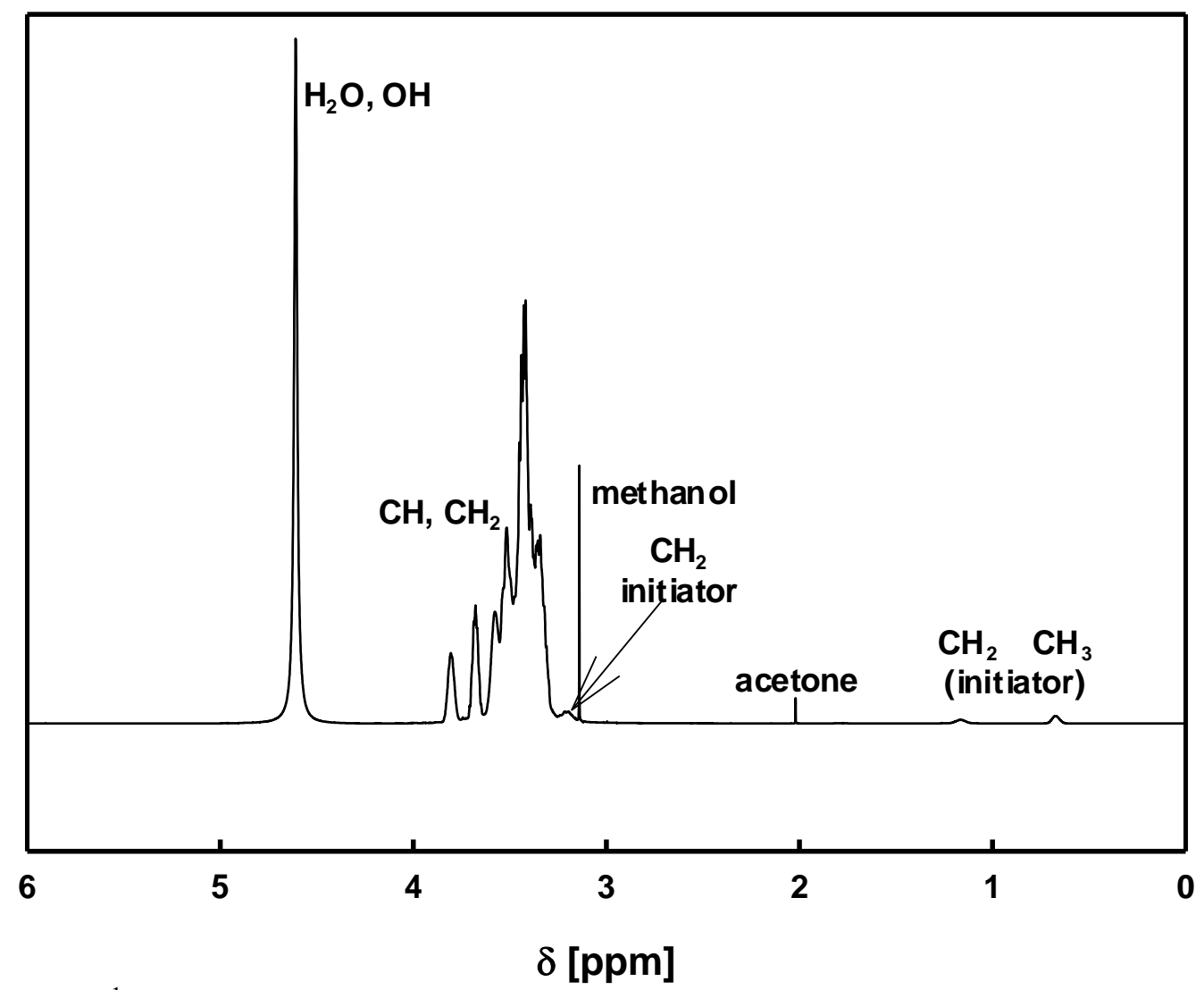

Figure S-2. ${ }^{1} \mathrm{H}$ NMR spectrum of hyperbranched polyglycidol used for hydrogel formation. 

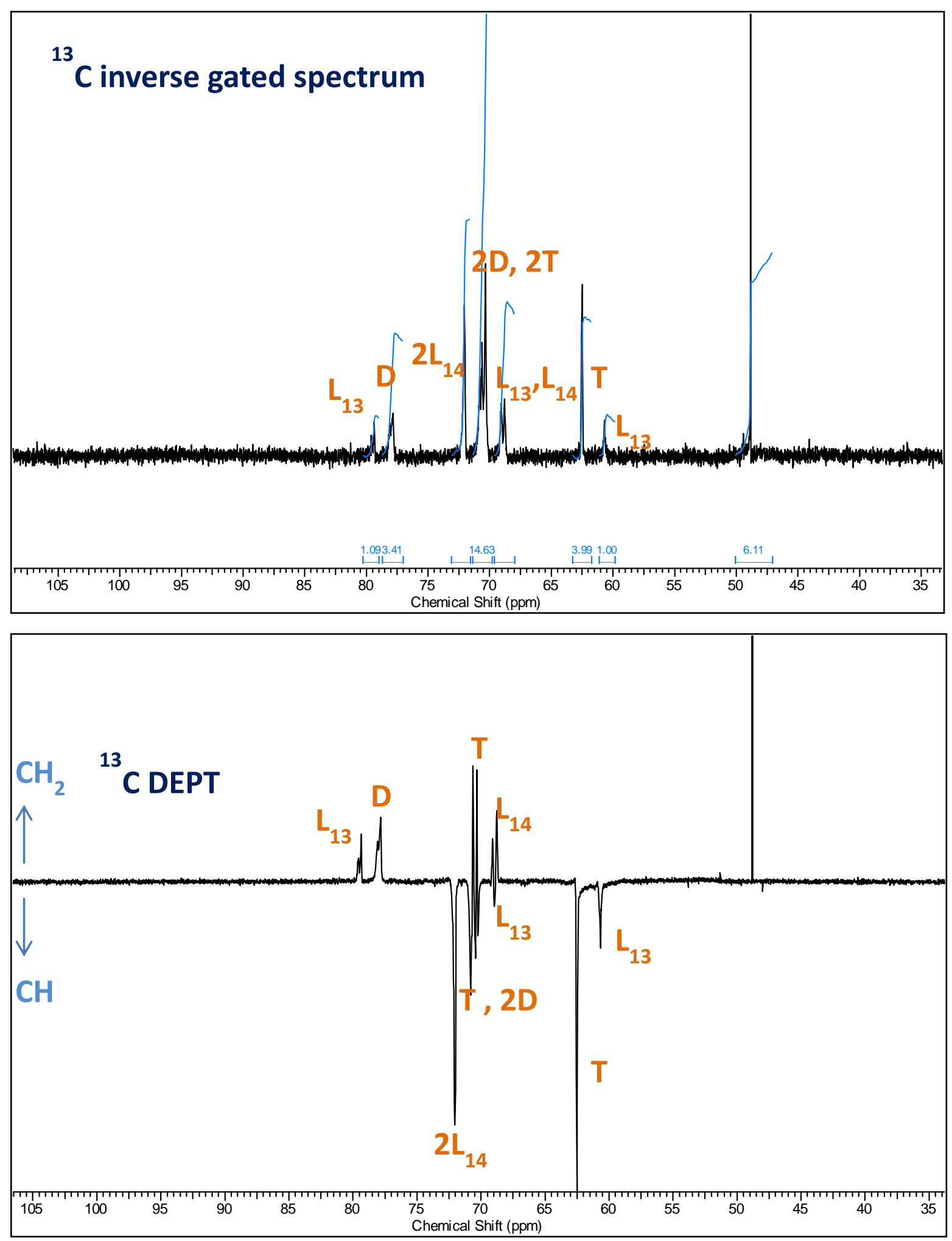

Figure S-3. ${ }^{13} \mathrm{C}$ NMR spectra of hyperbranched polyglycidol. At the top ${ }^{13} \mathrm{C}$ inverse gated spectrum. At the bottom DEPT spectrum. T, D, $\mathrm{L}_{13}, \mathrm{~L}_{14}$ denotes carbons belonging to the terminal, dentritic, linear 1,3 and linear 1,4 units, respectively, according to literature (A. Sunder, R. Hanselmann, H. Frey, R. Mülhaupt, Macromolecules 1999, 32, 4240-4246). 


\section{SI.2. Characteristics of hydrogel systems}

SI.2.1. Solid-state ${ }^{11}$ B NMR measurements of hydrogels at various temeperatures

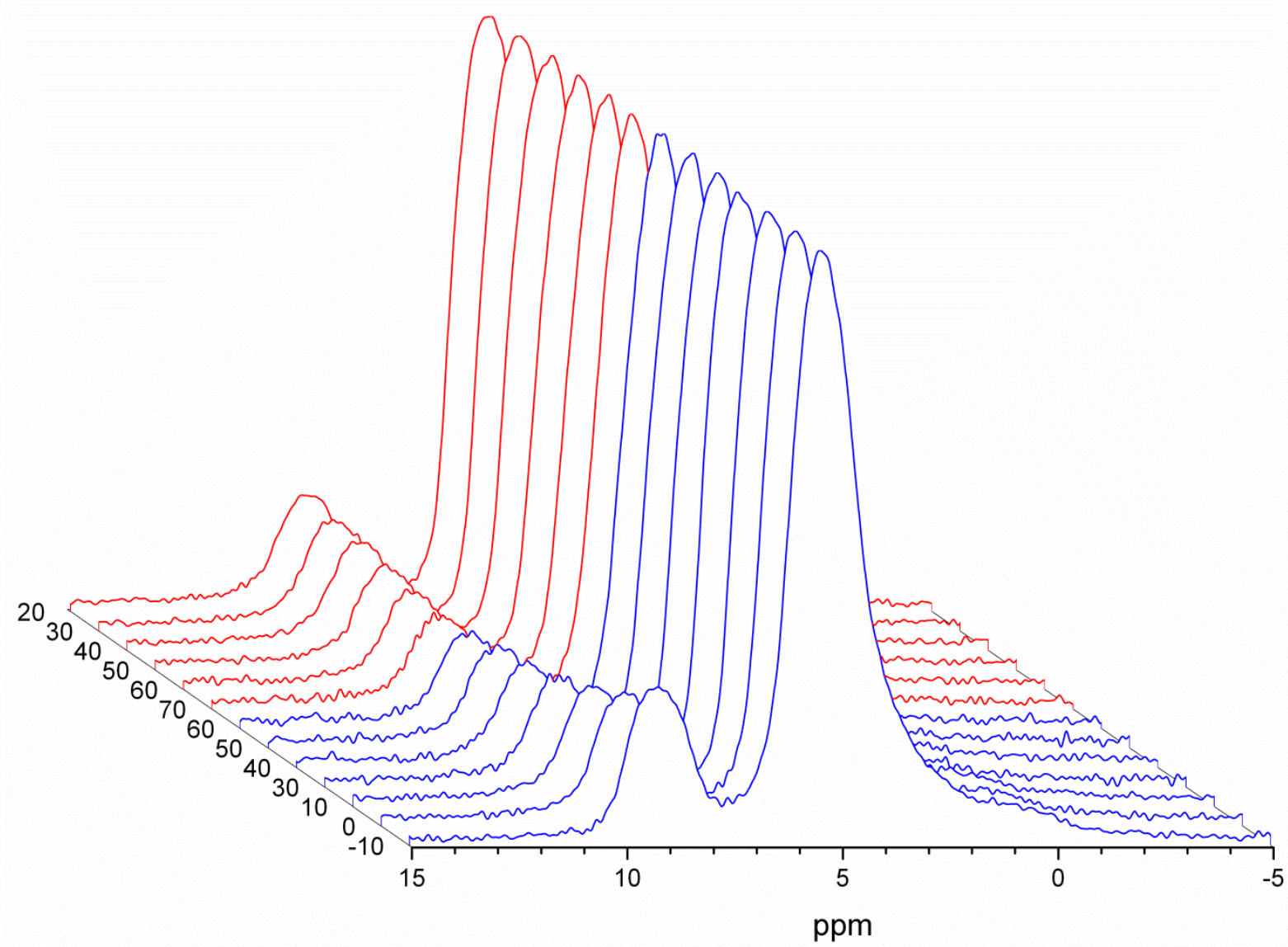

Figure S-4. Solid-state ${ }^{11}$ B NMR spectra of hydrogel H1 recorded at various temperatures. Red spectra correspond to gradual heating the hydrogel from $20^{\circ} \mathrm{C}$ to $60^{\circ} \mathrm{C}$, blue spectra gradual cooling the hydrogel from $50^{\circ} \mathrm{C}$ to $-10^{\circ} \mathrm{C}$. 


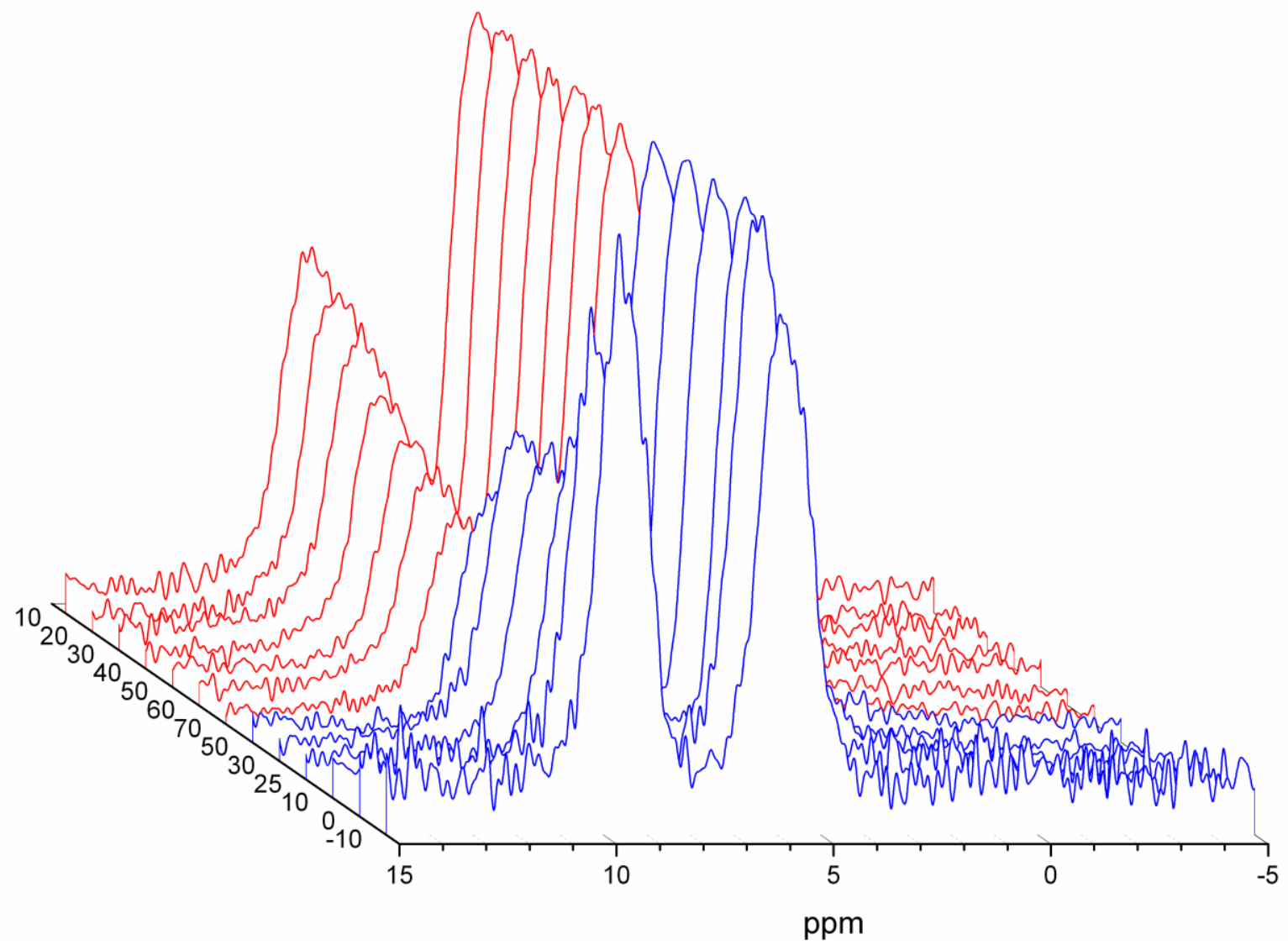

Figure S-5. Solid-state ${ }^{11}$ B NMR spectra of hydrogel $\mathrm{H} 2$ recorded at various temperatures. Red spectra correspond to gradual heating the hydrogel from $10^{\circ} \mathrm{C}$ to $70^{\circ} \mathrm{C}$, blue spectra gradual cooling the hydrogel from $50^{\circ} \mathrm{C}$ to $-10^{\circ} \mathrm{C}$.

\section{SI.2.2. DSC analyses of hydrogels}

Calorimetric studies of hydrogels were performed using Differential Scanning Calorimetry (DSC PYRIS Diamond Perkin Elmer, USA). The weighed samples were sealed in aluminum pans. An inert atmosphere surrounding the samples during the measurements was maintained using He flow. To avoid the white frost of external elements of the system, the purging gas $\left(\mathrm{N}_{2}\right)$ was used. Calibration for heat flux and temperature was done with indium and water. The studies of freezing and melting of the samples were performed in the range of phase transition with a linear temperature change rate of $10^{\circ} \mathrm{C} / \mathrm{min}$. 


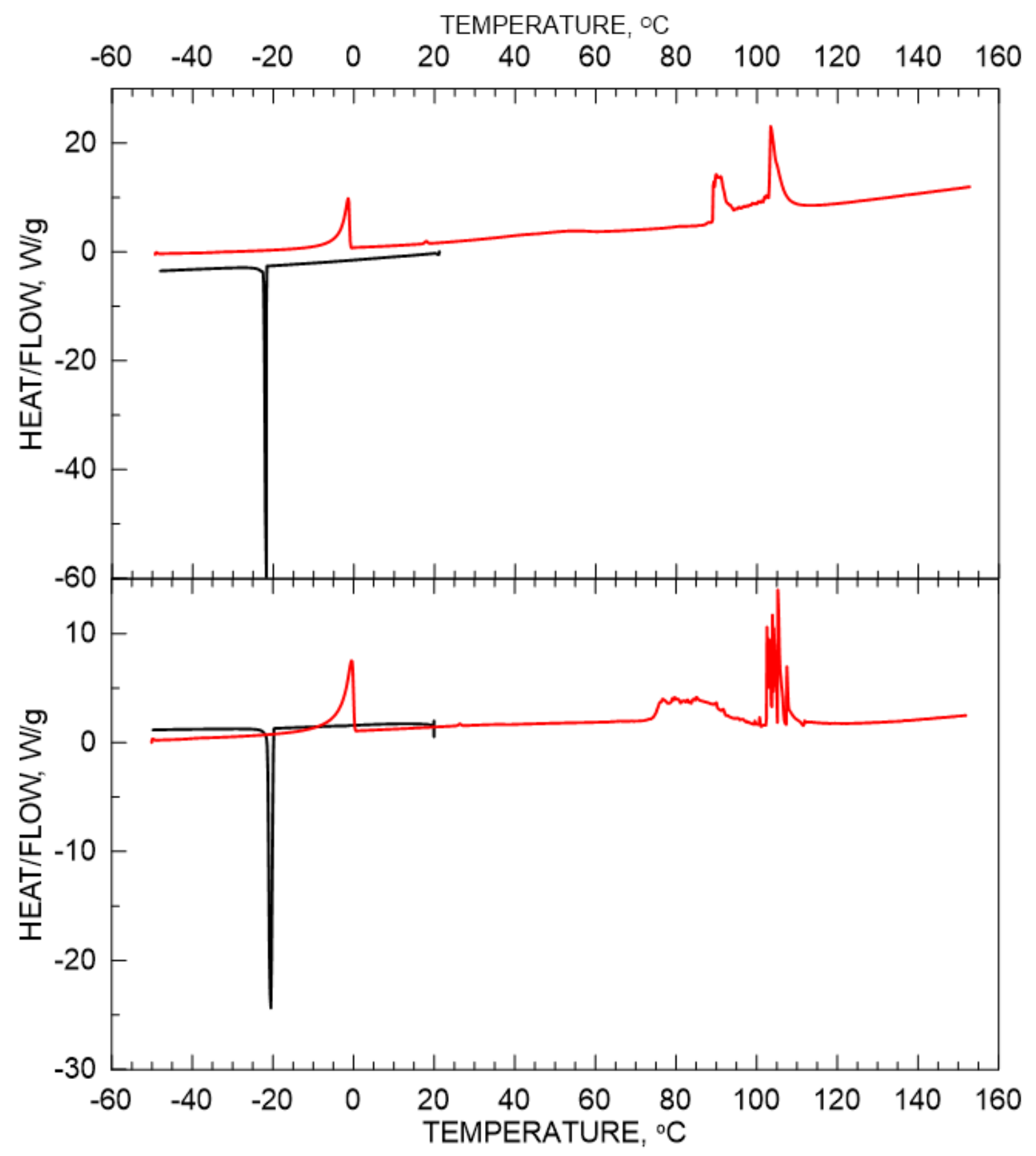

Figure S-6. DSC analyses carried out for hydrogels H1 (at the top) and H2 (at the bottom). Black and red lines denote cooling and heating cycles, respectively. 